\title{
Muerte cerebral secundaria a intoxicación por metanol
}

\section{Brain death secondary to methanol poisoning}

\author{
Ángel Alberto Garcia, Leonar G. Agiar, Juliana Granada • \\ BOgotÁ, D.C. (COLOMBIA)
}

\section{Resumen}

Las intoxicaciones por alcohol metílico son raras, pueden ser secundarias a intentos suicidas, accidentes e incluso a envenenamientos endémicos, tienen consecuencias severas que incluyen la muerte, secundarias a productos de la degradación del alcohol metílico por la alcohol deshidrogenasa, a continuación, presentamos un caso de intoxicación por alcohol metílico que como secuelas presentó alteraciones neurológicas y muerte. (Acta Med Colomb 2012; 37: 211-214)

Palabras claves: metanol, etilenglicol, intoxicación aguda, fomepizol, hemorragia intraparenquimatosa.

\begin{abstract}
Methyl alcohol poisoning is rare and may be secondary to suicide attempts, accidents and is also caused by endemic poisonings. It has severe consequences including death secondary to degradation products of methyl alcohol by alcohol dehydrogenase. We present a case of methyl alcohol poisoning that presented neurological alterations and death. (Acta Med Colomb 2012; 37: 211-214)

Keywords: methanol, ethylene glycol, acute poisoning, fomepizole, intraparenchymal hemorrhage
\end{abstract}

\author{
Dr. Ángel Alberto García Peña: Especia- \\ lista en Medicina Interna y Cardiología \\ Hospital Universitario San Ignacio. Pon- \\ tificia Universidad Javeriana; Dr. Leonar \\ G. Aguiar M.: Especialista en Medicina \\ Interna, Candidato a Maestría en Bioética, \\ Pontificia Universidad Javeriana. Bogotá, \\ D.C. Advanced Fellowship in Emergency \\ Medicine, George Washington University; \\ Dra. Juliana Granda Romero: Residente \\ de $2^{\circ}$ Año de Medicina de Emergencias. \\ Hospital Universitario San Ignacio, Ponti- \\ ficia Universidad Javeriana. Bogotá, D.C \\ (Colombia). \\ Correspondencia. Dra. Juliana Granada \\ Romero \\ E-mail: jgranada@javeriana.edu.co \\ Recibido: 20/XI/2011 Aceptado: 25/X/2012
}

\section{Caso clínico}

Paciente de sexo masculino de 30 años de edad, sin antecedentes patológicos conocidos, con antecedente de consumo frecuente de sustancias psicoactivas (marihuana/ cocaína), tres veces a la semana, quien ingresa al hospital universitario San Ignacio posterior a ingesta de alcohol etílico y metílico este último de forma accidental presentando ocho horas más tarde delirio, inapetencia, mareo y sed seguido de mayor alteración del estado de consciencia, sin adecuada respuesta a estímulos, con habla incoherente por lo que sus compañeros deciden asistir al servicio de urgencias. Durante el traslado se torna más agitado, con gritos inmotivados, actitud ansiosa, mayor lenguaje incoherente, desviación de la mirada y emesis.

$\mathrm{Al}$ ingreso a urgencias se encuentra un paciente en malas condiciones generales, taquicárdico, polipneico, somnoliento, ansioso, con midriasis y lenguaje incoherente, durante observación presenta deterioro del patrón respiratorio y del estado de conciencia por lo que se decide realización de intubación orotraqueal y traslado a la unidad de cuidados intensivos, con sospecha de intoxicación por alcoholes (¿metanol?).

Posteriormente se reciben paraclínicos evidenciando gases arteriales con academia metabólica severa, hemograma normal, electrolitos sin alteraciones, función renal normal, acido láctico elevado, etanol $12 \mathrm{mg} / 100 \mathrm{ml}$, metanol positivo, canabinoides mayor $135 \mathrm{ng} / \mathrm{ml}$, resto de drogas de abuso negativas, TAC de cráneo con ligera dilatación de las astas occipitales de los ventrículos laterales que por los antecedentes tóxicos referidos puede ser secundario a atrofia central.

Se inicia manejo con alcohol etílico por SNG, con paraclínicos de control que evidenciaban persistencia de acidemia metabólica, con elevación de azoados, por lo que es valorado por nefrología, indicándose soporte dialítico con hemodiálisis con filtro 170x, QB de 300, QD de 700, UF 0 sin heparina por cuatro horas.

Se tomaron gases arteriales de control al finalizar la primera hemodiálisis encontrando gases arteriales normales sin alteración del equilibrio ácido base, apesar de estabilización del $\mathrm{pH}$, el paciente presenta evolución tórpida, deterioro neurológico dado por ausencia de reflejos de tallo por lo que se realiza tomografía de cráneo simple de control, la cual evidencia hemorragia intraparenquimatosa, con signos de herniación, realizándosele doppler transcraneal (Figura 1) con el cual se exploraron las arterias cerebrales medias por vía transtemporal obteniendo curvas espectrales con morfología "en espiga", sin flujo neto hacia el parénquima cerebral, hallazgo compatible con muerte cerebral, siendo 
valorado en dos ocasiones por grupos multidisciplinarios diferentes que incluían cuidado intensivo, neurología y neurocirugía, presentando hipotensión progresiva y muerte 12 horas más tarde

\section{Discusión}

El alcohol metílico es un líquido volátil e incoloro, con olor y sabor desagradable, pero con destilación adicional se torna casi inodoro e insaboro, no tiene unión a proteínas, la dosis letal en humanos es de $0.5-1 \mathrm{~g} / \mathrm{kg}$ (1). El metanol es conocido como el alcohol de madera ya que fue destilado de la madera por primera vez en 1920, actualmente casi todo el metanol es hecho sintéticamente por medio de reducción catalítica del monóxido de carbono o dióxido de carbono en presencia de hidrógeno (2).

El alcohol metílico se absorbe por todas las vías, por vía gastrointestinal tiene una vida media de absorción de cinco minutos con un pico de absorción entre 30 a 60 minutos. Este se absorbe bien a través de la piel, por vía inhalatoria tiene una vida media de absorción de 0.80 horas; la tasa de absorción pulmonar es de 65-75\%. Por ser hidrosoluble difunde a todos los órganos ricos en agua como el cerebro, riñones, humor acuoso $(3,4)$.

El metanol por sí solo es poco tóxico, la toxicidad del metanol es causada por su metabolismo el cual es el encargado de la transformación a metabolitos tóxicos (5). Este ocurre principalmente en el hígado donde se metaboliza 90\% del alcohol metílico por medio de la alcohol deshidrogenasa (ADH) que se encarga de oxidar el metanol a formalaldehído, el cual posteriormente sufre una nueva oxidación por la formaldehídodeshidrogenasa y se convierte en ácido fórmico, el ácido fórmico se convierte luego a dióxido de carbono por medio de agua y 10 -formil tetrahidrofolato sintetasa; el ácido fórmico se acumula en la intoxicación por metanol y sus concentraciones están directamente relacionadas con la morbimortalidad $(6,7)$ (Figura 2). El ácido fórmico afecta el sistema nervioso central, particularmente el nervio óptico, además inhibe la citocromo oxidasa y con ella el proceso de respiración mitocondrial, llevando a disfunción hipóxica celular, este proceso es el causante de la mayor acidosis metabólica con anión gap elevado, el resto corresponden a producción de lactato, butirato y acetato (8).

La principal toxicidad se da por injuria ocular, la cual se produce en la parte retrolaminar del nervio óptico y en la retina, así como en el SNC específicamente a nivel de los ganglio basales (9).

Las manifestaciones clínicas dependen del tiempo entre la ingesta y la consulta y se pueden identificar dos periodos (Tabla 1).

\section{Periodo de latencia}

Ocho-24 horas (promedio de 12), puede prolongarse hasta dos días después de la exposición. No presentan sintomatología específica, frecuentemente se confunde y es poco diferenciable de la intoxicación etílica. El paciente

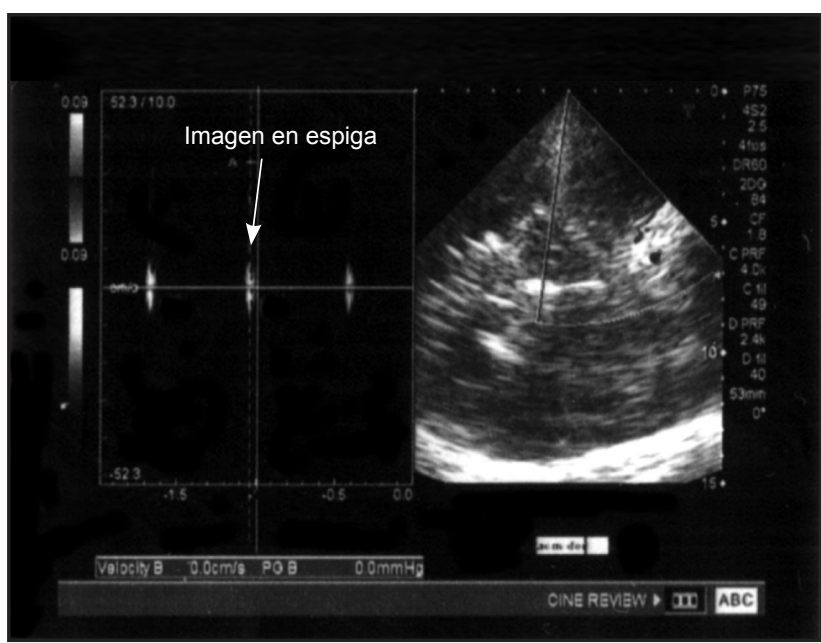

Figura 1. Exploración con Doppler triplex de las arterias cerebrales medias por vía transtemporal, obteniendo curvas espectrales con morfología "en espiga" sin flujo neto hacia el parénquima cerebral, hallazgo compatible con arresto cerebral.

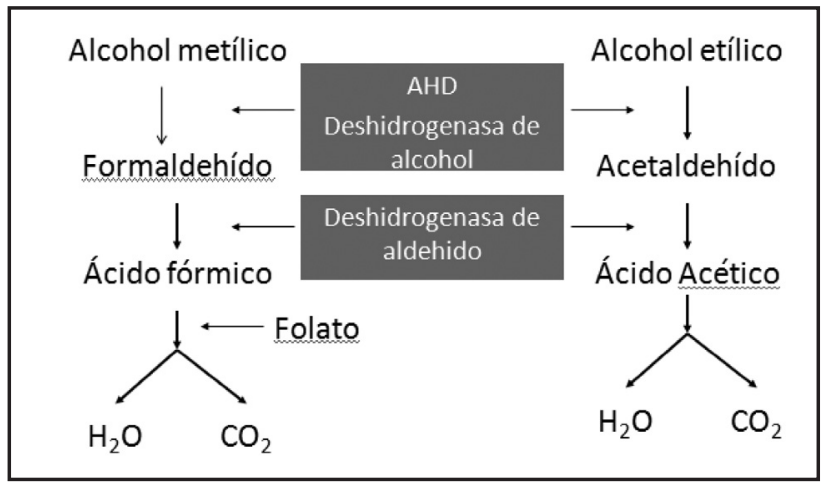

Figura 2 Proceso metabólico del alcohol metílico y etílico con sus diferentes reacciones.

Tabla 1. Manifestaciones clínicas de la intoxicación por alcohol metílico en relación con el grado de intoxicación y el valor de metanol en sangre.

\begin{tabular}{|c|l|l|}
\hline Metanol mg/100cc & Grado & \multicolumn{1}{c|}{ Clínica } \\
\hline$<10$ & Leve & $\begin{array}{l}\text { Náuseas, epigastralgia, cefalea, alteraciones } \\
\text { visuales de percepción o acomodación, } \\
\text { hiperemia de papila. }\end{array}$ \\
\hline $10-50$ & Moderada & $\begin{array}{l}\text { Vómito, embriaguez, diaforesis, visión } \\
\text { borrosa, taquipnea, disminución del reflejo } \\
\text { fotomotor, edema de papila. }\end{array}$ \\
\hline$>50$ & Severa & $\begin{array}{l}\text { Agitación, delirium, convulsiones, coma, } \\
\text { respiración de Kussmaul, cianosis, hipoten- } \\
\text { sión, muerte. }\end{array}$ \\
\hline
\end{tabular}

presenta síntomas de embriaguez, percibidos como un "guayabo" más intenso de lo usual, presentando cefalea pulsátil, dolor abdominal, náuseas, vómito, incoordinación motora y depresión leve del sistema nervioso central. En este periodo generalmente no se presenta acidosis metabólica, ya que el metabolismo del metanol no es tan rápido.

Este periodo puede prolongarse cuando se ha ingerido etanol simultáneamente $(5,10)$. 


\section{Periodo de acidosis metabólica}

Se presenta 12-30 horas después de la ingestión de alcohol metílico. Los pacientes tienen gran variedad de síntomas, entre los cuales se destacan cefalea, náuseas, vómito, dolor abdominal tipo cólico, mialgias y diarrea en menor proporción, además el paciente puede presentar dificultad respiratoria con taquipnea (respiración de Kussmaul), bradicardia e hipotensión, alteraciones del sistema nervioso como parestesias, debilidad muscular y convulsiones

Si el paciente no recibe tratamiento, el cuadro progresa y se presenta ceguera, choque, coma, convulsiones, persistencia de acidosis, anuria, colapso circulatorio, y finalmente muerte la cual generalmente es secundaria a falla respiratoria seguida de paro respiratorio $(11,12)$.

A nivel del sistema nervioso central lo más común es encontrar necrosis bilateral del putamen, que puede ser hemorrágica o necrótica $(13,14)$. Los mecanismos por lo que ésta se produce continúan siendo desconocidos, se cree que es uno de los lugares con mayor demanda metabólica llevando a aumentar la acumulación de acido fórmico en este lugar; otro mecanismo sugerido es la disminución del flujo sanguíneo a través de las venas basales de Rosenthal $(15,16)$. Los hallazgos característicos en el TAC son de baja atenuación y sólo son visibles en los pacientes que sobreviven más de 24 horas, en la intoxicación aguda el TAC generalmente es normal (14). Los pacientes que sobreviven a esta primera fase de toxicidad del sistema nervioso central generalmente desarrollan síntomas extrapiramidales (17). Niveles de metanol en relación con la gravedad de la intoxicación y la clínica.

En los paraclínicos normalmente se evidencia acidosis metabólica severa con aumento del anión gap, cuando las intoxicaciones son más severas normalmente el bicarbonato se encuentra menor de $18 \mathrm{mEq} / \mathrm{l}$, lo cual nos habla de concentraciones de metanol mayores a $50 \mathrm{mg} / \mathrm{dl}(500$ $\mathrm{mg} / \mathrm{l}$ ), por lo cual todos los pacientes en quienes se sospecha intoxicación por metanol deben tener gases arteriales, ya que la mortalidad tiene una mayor relación con la acidosis que con los niveles séricos de metanol $(2,18)$.

Los exámenes de rutina en intoxicaciones graves, deben incluir niveles séricos de metanol y etanol, electrolitos completos para cálculo de anión gap y osmolaridad, hemograma, nitrogenados, uroanálisis, enzimas hepáticas y amilasa (2). Es necesario recordar que el aumento de la amilasa no necesariamente implica que el paciente está cursando con pancreatitis, sin embargo si ésta se presenta normalmente se asocia a pancreatitis necrotizante severa (19).

En cuanto a imágenes, la más comúnmente solicitada es la tomografía, la cual puede ser normal cuando se toma en las primeras 24 horas, los hallazgos que más se encuentran son necrosis bilateral del putamen, en los casos de intoxicación severa con coma asociado se evidencia hipodensidad en el putamen y menos comúnmente en el núcleo caudado (20).

$\mathrm{Al}$ igual que con cualquier paciente intoxicado o con sobredosis el manejo inicial en el servicio de urgencias debe ir dirigido a manejar y proteger la vía aérea, si es necesario realizar intubación orotraqueal para asegurar una adecuada ventilación, y verificar una circulación efectiva (5).

Aun si el paciente consulta tempranamente debido a la rápida absorción del metanol, el lavado gástrico no es efectivo, por lo tanto la recomendación mundial es no realizarlo $(2,21)$.

En pacientes en quienes se encuentre $\mathrm{pH}$ menor a $7.3 \mathrm{se}$ debe considerar intoxicación grave (Tabla 2) y deben ser tratados con bicarbonato de sodio para corregir la acidosis ya que esto ayuda a disminuir la conversión de acido fórmico a formato.

El tratamiento definitivo debe estar dirigido a reducir al máximo la formación de los metabolitos tóxicos del metanol, lo cual se logra con la administración de etanol o fomepizol (22).

El etanol tiene aproximadamente 10 veces más afinidad por la alcohol deshidrogenasa que el metanol, por lo tanto inhibe competitivamente el metabolismo del metanol a sus metabolitos tóxicos (2). El fomepizol ha demostrado ser un potente inhibidor de la alcohol deshidrogenasa en micos y humanos, concentraciones en plasma de 0.8 $\mathrm{mg} / \mathrm{l}$ ha demostrado inhibir la alcohol deshidrogenasa en micos (23).

No hay ningún estudio que demuestre mayor efectividad de fomepizol sobre el alcohol etílico, su mayor desventaja es el alto costo lo cual disminuye su utilización; sin embargo, se prefiere el uso de fomepizol por varias razones: es más fácil de administrar que el etanol, tiene una duración de acción mayor, no causa depresión del sistema nervioso central, su administración no requiere monitoreo en cuidado intensivo, no produce hipoglicemia en pacientes predispuestos como en niños y en mujeres embarazadas, no produce alteraciones fetales (2).

El etanol es absorbido rápidamente por vía gastrointestinal, principalmente en el duodeno, por tanto los factores que prolongan el vaciamiento gástrico pueden retardar la absorción del etanol. El etanol cruza rápidamente la barrera hematoencefálica y placentaria, entre $90-98 \%$ es metabolizado en el hígado y el resto es excretado por los riñones y la respiración, la tasa de eliminación es de $15-20 \mathrm{mg} / \mathrm{dL} / \mathrm{h}$ (150-200 mg/L/h) (24).

La dosis inicial de etanol es de 600-800 mg/kg, lo cual corresponde a $1.8 \mathrm{ml} / \mathrm{kg}$ vía oral de alcohol a $43 \%$ o $7.5 \mathrm{ml} /$ $\mathrm{kg}$ de alcohol endovenoso al $100 \%$ seguida de una dosis

Tabla 2. Indicaciones para manejo con bicarbonato de sodio en intoxicaciones con alcohol metílico.

\begin{tabular}{|l|}
\hline Concentración de metanol mayor a $20 \mathrm{mg} / \mathrm{dl}(200 \mathrm{mg} / \mathrm{l})$ \\
\hline Historia de consumo sin conocer cantidad ni concentración pero con anion gap mayor \\
a $10 \mathrm{mOsm} / \mathrm{kg} \mathrm{H} 2 \mathrm{O}$ \\
\hline Sospecha clínica de ingesta de metanol con alguno de los siguientes criterios \\
1. Ph menor 7.3 \\
2. $\mathrm{HCO}_{2}$ menor $20 \mathrm{mEq} / \mathrm{L}(\mathrm{moml} / \mathrm{L})$
\end{tabular}


de mantenimiento de $66-154 \mathrm{mg} / \mathrm{kg} / \mathrm{hora}$, esta dosis varía dependiendo de la concentración de alcohol que se este administrando, correspondiendo a $0.2-0.46 \mathrm{ml} / \mathrm{kg} / \mathrm{h}$ vía oral con alcohol al $43 \%$, en caso de contarse con etanol intravenoso al $100 \%$ la dosis es de $1.96-2.13 \mathrm{ml} / \mathrm{kg} / \mathrm{h}$. La dosis de mantenimiento se debe ajustar en pacientes quienes se encuentran en diálisis a $169-257 \mathrm{mg} / \mathrm{kg} / \mathrm{h}$, que corresponde a $0.5-0.77 \mathrm{ml} / \mathrm{kg} / \mathrm{h}$ de alcohol via oral al $43 \%$, o $2.13-3.26$ $\mathrm{ml} / \mathrm{kg} / \mathrm{h}$ de alcohol endovenoso al 100\% (25).

Los niveles de etanol deben ser monitoreados cada dos horas para verificar que las concentraciones en sangre se encuentren entre $100-150 \mathrm{mg} / \mathrm{dl}(2)$.

Se recomienda diluir $50 \mathrm{cc}$ de etanol al $96 \%+450 \mathrm{cc}$ de DAD al 5\% para llevar a concentraciones al 10\% (1 ml $=100 \mathrm{mg}$ ) y administrar en niños dosis inicial de $5 \mathrm{cc} / \mathrm{kg}$ y en adultos $10 \mathrm{cc} / \mathrm{kg}$ en bolo inicialmente y continuar con una infusión de $1 \mathrm{cc} / \mathrm{kg} / \mathrm{h}$ hasta 24 horas después de desaparecer los niveles en sangre de metanol (5).

De no contarse con etanol IV, se podrán usar bebidas con contenido de alcohol entre 30 y $45 \%$ por vía oral a dosis en niños de $2 \mathrm{cc} / \mathrm{kg}$ cada tres horas y en adultos $30 \mathrm{cc} / \mathrm{kg}$ cada dos a tres horas (2).

El etanol puede causar hipoglicemia, principalmente en niños y malnutridos, la administración del etanol puede causar síntomas de intoxicación por este alcohol, además la presentación IV al 10\% puede causar flebitis por ser hiperosmolar, por lo que se recomienda usar una vía venosa central.

El fomepizol se absorbe rápidamente por vía oral y gastrointestinal y se distribuye rápidamente en toda el agua corporal, $97 \%$ de su eliminación es hepática, tiene varios metabolitos pero el principal es el 4-carboxipirazol, responsable de $80-85 \%$ de la acción terapéutica, su eliminación ocurre en 30-40 horas posterior a su administración.

La dosis recomendada es un bolo de $15 \mathrm{mg} / \mathrm{kg}$ seguido de $10 \mathrm{mg} / \mathrm{kg}$ cada 12 horas por cuatro dosis, para mantener niveles de fomepizol mayores a $0.8 \mathrm{mg} / \mathrm{L}$, durante diálisis la dosis de mantenimiento es de $1-1.5 \mathrm{mg} / \mathrm{kg} / \mathrm{h}$.

Los efectos adversos más comunes con fomepizol son náuseas, discinesia y cefalea $(26,27)$.

La hemodiálisis debe ser considerada en pacientes con acidosis metabólica severa con $\mathrm{pH}$ 7.25-7.30, con anormalidades visuales, deterioro de los signos vitales a pesar de manejo indicado, falla renal, alteraciones electrolíticas, no respuesta a manejo convencional, ingestión de más de 30 $\mathrm{ml}(0.5 \mathrm{ml} / \mathrm{kg}$ en niños $)$ de metanol, niveles de metanol en sangre $>50 \mathrm{mg} / \mathrm{dl}$, brecha osmolar $>10 \mathrm{mmol} / \mathrm{L}(28)$.

\section{Financiación y conflictos de interés}

Este artículo no recibió ningún tipo de financiamiento, su publicación es apoyada por la Pontificia Universidad Javeriana, Ninguno de los autores tiene conflictos de interés ni ha recibido apoyo de la industria farmacéutica

\section{Agradecimientos}

Agradecemos a los docentes y residentes que colaboraron en el caso.

\section{Referencia}

1. Davis LE, Hudson D, Benson BE, Jones Easom LA, Coleman JK. Methanol Poisoning Exposures in the United States: 1993-1998. Clinical Toxicology 2002; 40(4): 499-505

2. Barceloux DG, Bond GR, Krenzelok EP, et al. American Academy of Clinica Toxicology Practice Guidelines on the Treatment of Methanol Poisoning. Clin Toxicol 2002; 40: 415-46.

3. Graw M, Haffner HT, Althaus L, Besserer K, Voges S. Invasion and Distribution of Methanol. Arch Toxicol 2000; 74: 313-321.

4. Osterloh JD, D'Alessandro A, Chuwers P, Mogadeddi H, Kelly TJ. Serum concentrations of methanol after inhalation at $200 \mathrm{ppm}$. J Occup Environ Med 1996; 38: 571-576.

5. Williams GF, Hatch FJ. Methanol poisoning: a review and case study of four patient from Central Australia. Aust Crit Care 1997; 10(4): 113-8.

6. Jacobsen D, McMartin KE. Methanol and ethylene glycol poisonings. Mechanims of toxicity, clinical course, diagnosis and treatmen. Med Toxicol 1986 1(5): 309-334.

7. Allister Vale. Methanol. Poisonous substances. 2003. The Medicine Publishing Company Ltd.

8. Chiang WK. Alcohols. Emergency Medicine. 3rd edition 1992: 572-78.

9. White Suzanne R. Methanol, Rosen's Emergency Medicine: Concepts and Clinical Practice 5th edition 2002: 2127-2130.

10. Becker CE. Methanol puisontng. J Emerg Med 1983; 1(1): 51-58.

11. Skrzydlewska E. Toxicological and metabolic consequences of methanol poisoning. Toxicol Mech Methods 2003; 13: 277-93.

12. Hovda KE, Hunderi OH, Tafjord AB, et al. Methanol outbreak in Norway 2002-2004: epidemiology, clinical features and prognostic signs. J Intern Med 2005; 258: 181-90.

13. Singh A, Samson R, Girdhar A. Portrait of a methanol-intoxicated brain. Am J Med 2011; 124(2): 125-7.

14. Taheri MS, Moghaddam HH, Moharamzad Y, Dadgari S, Nahvi V. The value of brain CT findings in acute methanol toxicity. Eur J Radiol 2010; 73(2): 211-4.

15. Ferah Karayel, Arzu A. Turan, Aydin Sav, et al. Methanol intoxication: pathological changes of central nervous system (17 cases). Am J Forensic Med Pathol 2010; 31(1): 34-6.

16. Joaquín Serrano, Ángel Ríos, Natalia Fernández, Ana Aréval. Lesiones cerebrales secundarias a intoxicación por metanol. Galicia Clin 2010; 71(2): 59-60.

17. Fontenot AP, Pelak VS. Development of neurologic symptoms in a 26 -yearold woman following recovery from methanol intoxication. Chest 2002; 122: 1436-1439.

18. Swartz RD, Millman RP, Billi JE, et al. Epidemic Methanol Poisoning: Clinica and Biochemical Analysis of a Recent Episode. Medicine 1981; 60: 373-382.

19. Hantson P, Mahieu P. Pancreatic Injury Following Acute Methanol Poisoning. J Toxicol Clin Toxicol 2000; 38: 297-303.

20. Gaul HP, Wallace CJ, Auer RN, Fong TC. MR Findings in Methanol Intoxication. Am J Neuroradiol 1995; 16: 1783-1786.

21. Kruse J.A. Methanol Poisoning. Intensive Care Med 1992; 18: 391-397.

22. Becker CE. Methanol puisontng. J Emerg Med 1983; 1(1): 51-58.

23. McMartin KE, Hedström KG, Tolf BR. Studies on the Metabolic Interactions Between 4-Methylpyrazole and Methanol Using the Monkey as an Animal Model. Arch Biochem Biophys 1980; 199: 606-614.

24. Holford, N.H.G. Clinical Pharmacokinetics of Ethanol. Clin Pharmacokinet 1987; 13: 273-292.

25. American Academy of Clinical Toxicology Practice Guidelines on the Treatment of Methanol Poisoning. Clin Toxicol 2002; 40: 415-46.

26. Brent J, McMartin K, Phillips S, et al. Fomepizole for the Treatment of Ethylene Glycol Poisoning. N Engl J Med 1999, 340: 832-838.

27. Brent J; McMartin K, Phillips S, Aaron C, Kulig K. Fomepizole for the Treatment of Methanol Poisoning. N Engl J Med 2001; 344: 424-429.

28. Gonda A, Gault H. Churchill D, Hollomby D. Hemodialysis for Methanol Intoxication. Am J Med 1979, 64: 749-758. 